\title{
Diagnosing Dementia with Lewy Bodies: Current Challenges in the Clinical Setting
}

\section{Sivaraman Purushothuman*}

Brain \& Mind Centre and Central Clinical School, University of Sydney, NSW, Australia

*Corresponding Author: Sivaraman Purushothuman, Brain \& Mind Centre and Central Clinical School, University of Sydney, NSW, Australia.

Received: August 27, 2019; Published: September 30, 2019

DOI: 10.31080/ASNE.2019.02.0109

\begin{abstract}
Dementia with Lewy bodies (DLB) has been proven difficult to clinically differentiate from Alzheimer's disease (AD) and Parkinson's disease (PD). Unfortunately, autopsy confirmation remains the gold standard for accurate diagnosis of DLB. Consequently, DLB has been far less studied at both the genetic and mechanistic levels than AD and PD. Both DLB and AD share similar inheritance patterns and may also share some common risk factors and pathological features. However, DLB has distinct overall pathology manifesting in distinct early phenotypic patterns such as fluctuating cognition, pronounced visual and/or auditory hallucinations, together with movement disorders similar to parkinsonism. To understand DLB further, the other common and varied risk factors to AD and PD, and the associated cellular pathogenesis and genetic factors should be closely examined.
\end{abstract}

Keywords: Dementia with Lewy bodies (DLB); Diagnosis; Alzheimer's Disease (AD); Parkinson's Disease (PD)

\section{Dementia with Lewy bodies}

Dementia with Lewy bodies (DLB) is the second most common dementia syndrome representing 10 to $15 \%$ of total dementia cases, after Alzheimer's disease (AD) which accounts for the other $50-60 \%$ of all dementias [1]. DLB and Parkinson's disease with dementia (PDD) share the common neuropathological inclusions of Lewy body accumulation, and together, they are known as Lewy body dementias. Currently, considerable confusion exists concerning the clinical, neuropsychological, pathological and genetic delineation of DLB with AD, Parkinson's disease (PD) and PDD [2]. More accurate clinical diagnosis of DLB is important to determine the differences in prognosis and management for DLB from other dementing or movement disorders more efficiently. In general, DLB patients generally have a shorter survival period ( $\sim 2$ - 8 years) after reporting rapid declines in cognition and other domains during the clinical course after initial dementia diagnosis when compared to AD or PD patients [3].
A proportion (20 - 50\%) of patients with PD may develop dementia or PDD usually after 10 years following diagnosis [4]. Moreover, cognitive features of PDD are similar and often indistinguishable from the clinical syndrome of DLB, and there are only a few pathologic differences existing between DLB and PDDwith more severe neuronal loss in the substantia nigra in PDD than in DLB cases [5], whereas most DLB cases have AD-like amyloid pathology with some neurofibrillary tau tangles, while cortical amyloid load is infrequent in PDD [6,7]. When dementia develops after a year of an established motor disorder, we define it as PDD; in contrast, when dementia develops prior to or within one-year of motor deficits, we define the disease as DLB $[8,9]$.

Diagnostic criteria and pathophysiology for dementia with Lewy bodies

Third consensus report diagnostic criteria (2005)

Clinically, according to the diagnostic guidelines [2,8,10], DLB is characterised by progressive dementia, fluctuating cognition, 
recurrent visual hallucinations that are well-formed and detailed, fluctuating confusions, and associated extra-pyramidal parkinsonism-like symptoms. According to a meta-analysis study [11], the sensitivity, specificity and accuracy of the 2005 clinical diagnostic criteria [8] for DLB at late stage of disease was $84 \%, 67 \%$ and $78 \%$, respectively, giving rise to $20 \%$ clinical misdiagnosis [12]. It has also been reported that the overall diagnostic accuracy did not change over time [11] and this is dependent of the assessed populations [13]. Another previous study that assessed the 2005 DLB clinical diagnostic criteria presented with a low 32\% sensitivity for 'pure' DLB after systematically comparing final clinical diagnoses with subsequent neuropathology diagnoses [14]. Critically, the main challenge of clinical diagnosis in patients with DLB, PD and $\mathrm{AD}$ arises from the fact that the underlying pathology is frequently complex with contributions from both AD (Amyloid-beta and neurofibrillary tau tangles) and Lewy body disease pathologies $[8,13]$.

\section{Fourth (latest) consensus report diagnostic criteria (2017)}

Recently, a fourth consensus report of the DLB consortium [9] was released to revise the previous $3^{\text {rd }}$ consensus report, described above. This new criteria have incorporated a series of recommendations from the past decade to better describe DLB according to clinical settings and disease stages, such as the exclusion of antipsychotics for acute DLB symptom management and inclusion of REM sleep behaviour disorder as a core clinical feature. Also, the indicative imaging biomarkers (e.g. striatal $\left.{ }^{[123} \mathrm{I}\right]$ ioflupane SPECT dopamine transporter (DAT) and cardiac [123I] metaiodobenzylguanidine (MIBG) sympathetic myocardial scintigraphy) as an indirect way of measuring Lewy body pathology, carries some weight. At least one of these indicative biomarker together with at least one core clinical feature can now be used for a diagnosis of probable DLB. However, as two of the main features in the past and present criteria, visual hallucinations [15-17] and REM sleep behaviour disorder [18-20] were not differentiated to define the varied types and the timing of visual symptoms and sleep problems. As evident [21], confirmed that a significantly higher frequency of hallucinations, agitation and sleep problems was found in DLB versus AD or PDD.

\section{Conclusion}

Therefore, differentiating DLB from AD, PD and PDD may be a diagnostic challenge in clinical practice, even at any stages of disease. Further meta-analysis studies are warranted for clinical validity of the latest DLB consensus criteria.

\section{Acknowledgement}

This work is supported by the Australian Research Council (ARC) and National Health and Medical Research Council (NHMRC) Dementia Research Development Fellowship and grant awarded to S.P.

\section{Bibliography}

1. Boot BP., et al. "Risk factors for dementia with Lewy bodies: a case-control study". Neurology 81.9 (2013): 833-840.

2. Nervi A., et al. "Familial aggregation of dementia with lewy bodies”. Archives of Neurology 68.1 (2011): 90-93.

3. Larsson V., et al. "Relative survival in patients with dementia with Lewy bodies and Parkinson's disease dementia". PLoS One 13.8 (2018): e0202044.

4. Beyer K., et al. "The decrease of beta-synuclein in cortical brain areas defines a molecular subgroup of dementia with Lewy bodies". Brain 133.12 (2010): 3724-3733.

5. Tsuboi Y and DW Dickson. "Dementia with Lewy bodies and Parkinson's disease with dementia: are they different?" Parkinsonism and Related Disorders 11.1 (2005): S47-S51.

6. Edison P., et al. "Amyloid load in Parkinson's disease dementia and Lewy body dementia measured with [11C] PIB positron emission tomography". Journal of Neurology, Neurosurgery, and Psychiatry 79.12 (2008): 1331-1338.

7. Peraza LR., et al. “Resting state in Parkinson's disease dementia and dementia with Lewy bodies: commonalities and differences". International Journal of Geriatric Psychiatry 30.11 (2015): 1135-1146.

8. McKeith IG., et al. "Diagnosis and management of dementia with Lewy bodies: Third report of the DLB consortium". Neurology 65.12 (2005): 1863-1872.

9. McKeith IG., et al. "Diagnosis and management of dementia with Lewy bodies: Fourth consensus report of the DLB Consortium". Neurology 89.1 (2017): 88-100.

10. McKeith I. "Commentary: DLB and PDD: the same or different? Is there a debate?". International Psychogeriatrics 21.1 (2009): 220-224. 
11. Arcuti S., et al. "Accuracy of Clinical Diagnosis of Dementia with Lewy Bodies: A Systematic Review and Meta-Analysis". Journal of Neurology, Neurosurgery, and Psychiatry 89.4 (2016): 358-366.

12. Rizzo G., et al. "Accuracy of clinical diagnosis of dementia with Lewy bodies: a systematic review and meta-analysis". Journal of Neurology, Neurosurgery and amp; Psychiatry 89.4 (2018): 358-366.

13. Huang Y and G Halliday. "Can we clinically diagnose dementia with Lewy bodies yet?". Translational Neurodegeneration 2.1 (2013): 4 .

14. Nelson P., et al. "Low sensitivity in clinical diagnoses of dementia with Lewy bodies". Journal of Neurology 257.3 (2010): 359-366.

15. Wang G., et al. "Visual hallucinations and associated factors in Chinese patients with Parkinson's disease: roles of RBD and visual pathway deficit". Parkinsonism and Related Disorders 16.10 (2010): 695-696.

16. Postuma RB., et al. "Olfaction and color vision identify impending neurodegeneration in rapid eye movement sleep behavior disorder". Annals of Neurology 69.5 (2011): 811-818.

17. Armstrong R and H Kergoat. "Oculo-visual changes and clinical considerations affecting older patients with dementia". Ophthalmic and Physiological Optics 35.4 (2015): 352-376.

18. Ferman TJ., et al. "Inclusion of RBD improves the diagnostic classification of dementia with Lewy bodies". Neurology 77.9 (2011): 875-882.

19. Postuma RB., et al. "How does parkinsonism start? Prodromal parkinsonism motor changes in idiopathic REM sleep behaviour disorder". Brain 135.6 (2012): 1860-1870.
20. Postuma RB. "Prodromal Parkinson's disease - using REM sleep behavior disorder as a window". Parkinsonism and Related Disorders 20 (2014): 1-4.

21. Bougea A., et al. "Neuropsychiatric symptoms and $\alpha$-Synuclein profile of patients with Parkinson's disease dementia, dementia with Lewy bodies and Alzheimer's disease". Journal of Neurology 265.10 (2018): 2295-2301.

\section{Volume 2 Issue 10 October 2019}

(C) All rights are reserved by Sivaraman Purushothuman. 